\title{
Numerical simulation of melt convection in an AC electro-fused magnesia furnace for $\mathrm{MgO}$ production
}

\author{
Zhen Wang ${ }^{1, a}$, Ninghui Wang ${ }^{2, b}$ \\ ${ }^{1}$ School of Innovation and Entrepreneurship, Dalian University of Technology, Dalian 116024,China \\ ${ }^{2}$ School of Electrical Engineering, Dalian University of Technology, Dalian 116024,China \\ aueyou@sina.com, bninghuiw@263.net
}

Keywords: Fused MgO, Electric arc furnace, Convection, Simulation.

\section{Abstract.}

In order to better understand the melt convection in an electro-fused magnesia furnace (EFMF), a 3D finite element method based model of the furnace is presented. The effect of the electromagnetic stirring and buoyancy driving forces on the melt flow are investigated in three typical cases with different bath dimensions. Dimensionless parameters including Reynolds number, Richardson number and Prandtl number are introduced to characterize the melt convection. The numerical results indicate that the convection is fully turbulent, and the flow and temperature fields are both significantly influenced by the line current. According to the calculated Richardson number, neither nature nor forced convection is negligible in all cases. The buoyancy becomes more important when the bath volume grows and the current decreases, and the magnetic stirring becomes more important when the bath volume shrinks and the current increases.

\section{Introduction}

The arc fused MgO has a wide range of applications in modern science and technology, for it possesses better characteristics than the heavy burned magnesium oxide in chemical stability, moisture resistance and the electric insulation because of larger crystal grains. The arc fused MgO furnace (EFMF) is one of the main pieces of equipment used to produce arc fused MgO. The structure of EFMF is similar to the three-phase submerged arc furnace (SAF) for production of silicon metal and ferrosilicon. It is the principal object of EFMF to provide purified fused $\mathrm{MgO}$, and the melting point of $\mathrm{MgO}$ is much higher than most other materials in the metallurgical industry. Consequently, the technique is significantly different from other industries. Knowledge of the vortex structure of melt convection is important for technological optimization and improvement of MgO production. Using a specially designed SAF, Zhang et al. [1, 2] demonstrated the growth of cubic MgO single crystals with an average size of $6 \mathrm{~cm}$. A model describing the thermal behavior of the furnace for $\mathrm{MgO}$ production was used to select the best charge procedure under different conditions [3]. The between-mode process modeling and monitoring method of the EFMF is proposed to solve the control problems such as strong nonlinearity and multi-modes [4-6]. However, the investigations mentioned above didn't provide detailed information on the heat transfer and fluid flow phenomena. Caffery et al. [7] developed a model to conduct a temperature homogenization study for an electric arc furnace (EAF) steelmaking bath, but their investigation mainly focused on the influences of the oxygen lance position and bottom bubbles. Several 3D finite element models were used to calculate electromagnetic fields in an AC EAF [8-9]. However, the melt convection of the molten bath was not considered in these models. Numerical modeling has been used to investigate the influence of electromagnetic stirring on melting of a single piece of scrap in an EAF [10], but the melting materials were not MgO. The effect of the electromagnetic stirring in an EFMF was studied by Wang et al. [11] and it was found that the temperature field of the bath is significantly influenced by the melt flow. However, the flow pattern is only considered to be laminar.

The main aim of this work is to develop a mathematical model to investigate the relationship between the intensity of electric current and melt convection with the consideration of different bath shapes. 


\section{Experimental results}

The EFMF makes use of secondary-circuit three-phase alternating current applied to three electrodes that are arranged in a triangular configuration with a furnace shell. Fig. 1 shows schematic views of the horizontal and vertical cross sections of the EFMF. The furnace shell contains MgO raw materials and electrodes. One of the key issues in the melting processes is the control of the intensity of the line currents in order to achieve a better heating and purification effect. Fig. 2 shows the appearance of solidified MgO ingot. The size and shape of the ingot were significantly influenced by the power supplied in the melting process. The capacity of the EFMF transformer is $1500 \mathrm{kVA}$, and the frequency is $50 \mathrm{~Hz}$. The dimensions of the furnace are shown in Table 1 . The shape of the bath is determined mainly by the bath long $(\mathrm{Rl})$ and short (Rs) radiuses, and the bath depth $(\mathrm{H})$ which are shown in Fig. 1.

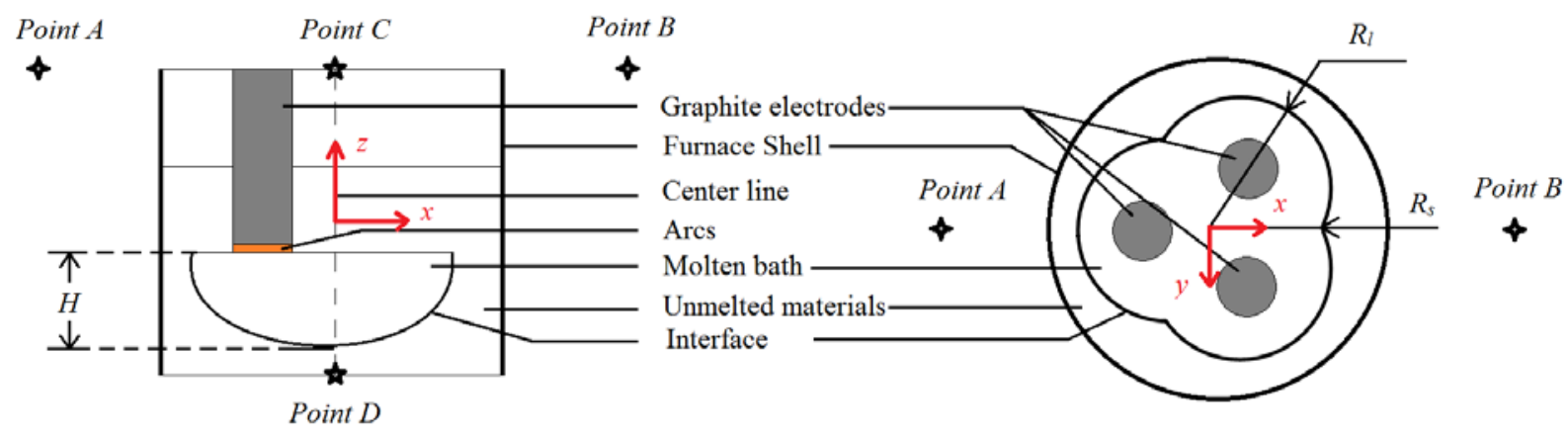

(a)

(b)

Fig. 1. Schematic views of the furnace: (a) the vertical cross section through the center line of the furnace and one of the electrodes; (b) the horizontal cross section of the furnace.

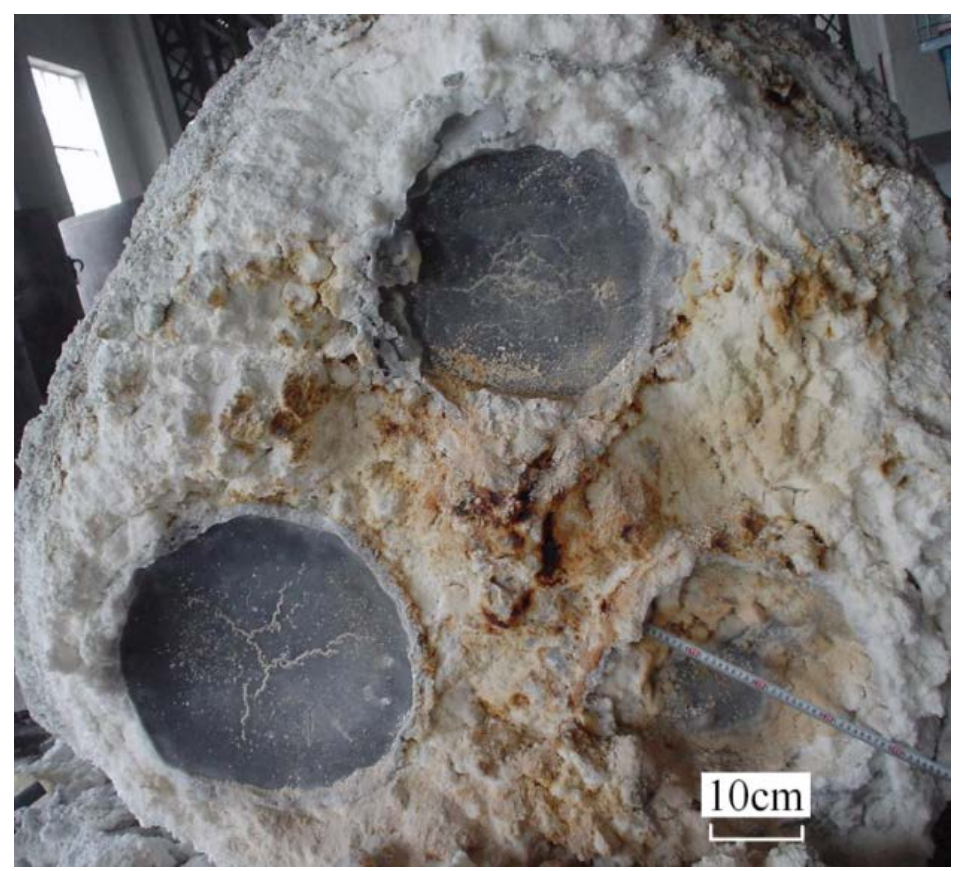

Fig. 2. The top view of the solidified MgO ingot

Table 1Dimensions of the model

\begin{tabular}{cc}
\hline Items & Value [mm] \\
\hline The furnace height & 2000 \\
The furnace diameter & 2000 \\
Electrode diameter & 300 \\
The distance between electrode centerlines & 650 \\
Electrode bottom depth & 1000 \\
\hline
\end{tabular}




\section{Results and discussion}

The numerical simulations of the electromagnetic stirring of the molten bath inside an EFMF show that the flow field is dependent on the bath shape and strength of the electrical current intensities. The shape of the molten bath is mainly decided by $R_{l}, R_{s}$, and $H$ which are defined in Fig. 2. $R_{l}$ and $R_{s}$ are the long and short radial distances from the center of the bath respectively. $H$ is the depth of the bath. The entire process could be divided into 3 cases. In Case 1, a small molten bath is formed when the arcs are just ignited, and the shape is characterized by $R_{l}=0.57 \mathrm{~m}, R_{s}=0.5 \mathrm{~m}$, and $H=0.5 \mathrm{~m}$; In Case 2 , a larger molten bath is formed when enough energy is consumed with the characteristic lengths of $R_{l}=0.77 \mathrm{~m}, R_{s}=0.667 \mathrm{~m}$, and $H=0.7 \mathrm{~m}$; In Case 3, a higher molten bath is formed with $R_{l}=0.77 \mathrm{~m}, R_{s}$ $=0.667 \mathrm{~m}$, and $H=1.0 \mathrm{~m}$. The line currents (RMS values) adopted in the following simulations are $10.6 \mathrm{kA}$, and $5.3 \mathrm{kA}$. Coupled-field analysis was performed by software ANSYS. The physical properties of MgO are taken from [12].

The average velocity of the melt flow driven by $10.6 \mathrm{kA}$ current is more than twice of that driven by 5.3kA. The values of Reynolds number are about 83200 (10.6kA) and 31200 (5.3kA) respectively within the bath, so the system is fully turbulent in Case 1. Consequently, the temperature difference of the melt system is less than $50 \mathrm{~K}$. Neither natural convection nor forced convection is negligible, because the scope of Richardson number varies from 0.77 (10.6kA) to 3.81 (5.3kA).

The similar situation also occurs for Case 2 shown in Fig. 3 . The volume of the bath become larger in this stage, and the Lorentz force density becomes smaller if the current level is the same. The average temperature and velocity both decrease comparing with the temperature and flow field in Case 1. A small vortex forms between the boundary of the bath and one electrode, for the flow space is large enough. The values of Reynolds number increase to 109200 (10.6kA) and 43680 (5.3kA) respectively. The scope of Richardson number changes from 1.1 (10.6kA) to 3.92 (5.3kA), and the larger values mean the influence of buoyancy increases with a larger bath radius.

The height $(H)$ increases based on the bath dimensions in Case 2, so the bath volume in Case 3 is the largest in all three cases. Consequently, the average density of volume power and Lorentz force are the lowest of all cases. The values of Reynolds number increase to 160160 (10.6kA) and 80080 (5.3kA). The scope of Richardson number changes from $1.85(10.6 \mathrm{kA})$ to $4.6(5.3 \mathrm{kA})$, and the larger values mean the influence of buoyancy increases with a larger bath height.

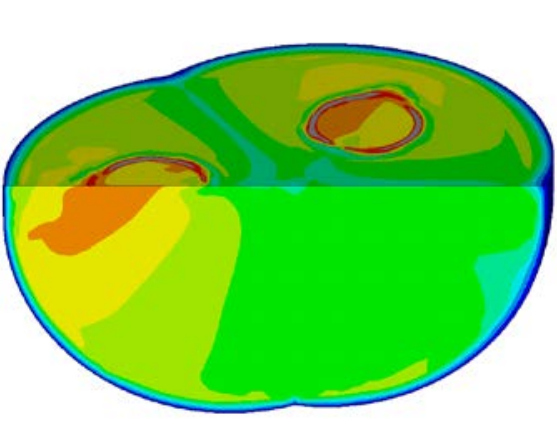

(a) Temperature field, $I=10.6 \mathrm{kA}$
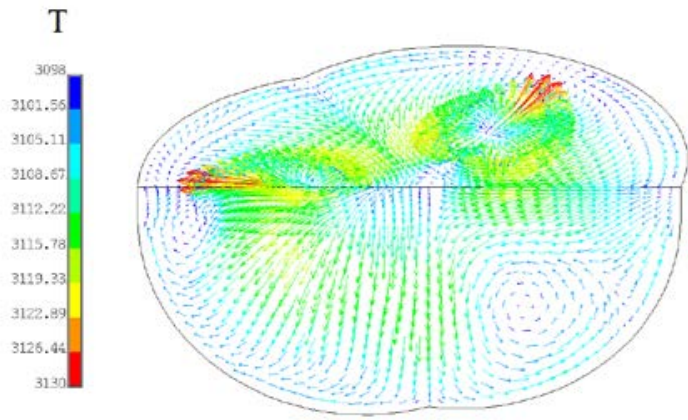

(b) Flow field, $I=10.6 \mathrm{kA}$

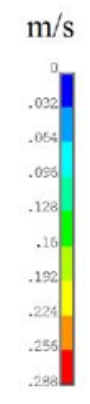




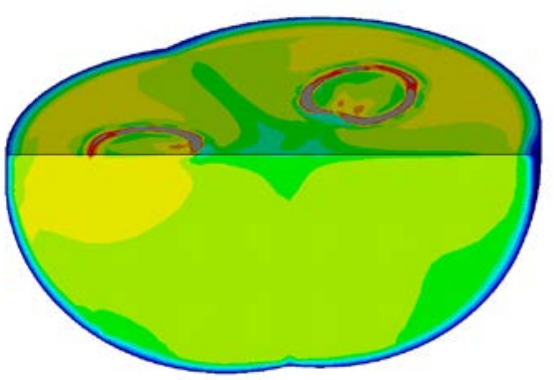

(c) Temperature field, $I=5.3 \mathrm{kA}$
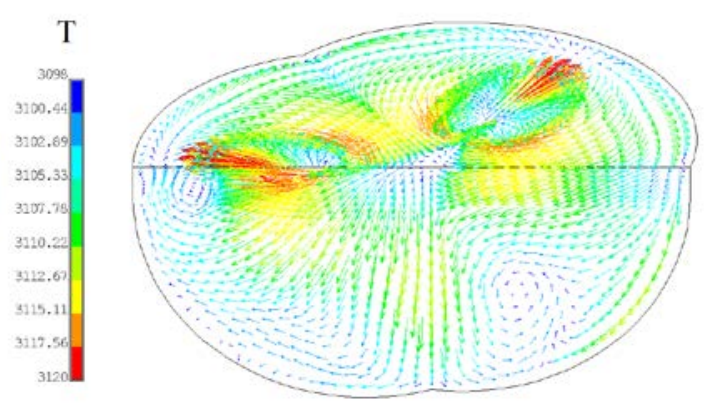

(d) Flow field, $I=5.3 \mathrm{kA}$

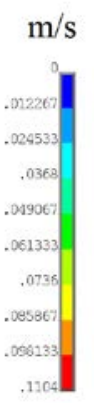

Fig. 3. The temperature and velocity distribution in Case 2 with the current of $10.6 \mathrm{kA}$ and 5.3kA. The molten bath is viewed along the line from point $\left(x=0, y=3^{1 / 2}, z=1\right)$ in the global coordinate system to the global coordinate system origin.

Fig. 4 shows that the Joule heating power (RMS) increases with the line current, and decreases with the bath volume. The Joule heating power reaches more than $300 \mathrm{kVA}$ with $10.6 \mathrm{kA}$ current in Case 1, and decreases to about 250kVA when the bath volume grows larger with the same current in Case 2 and 3 . The line resistance is nearly not influenced by the current, and it is mainly dependent on the bath volume. The value is about $3.1 \mathrm{~m} \Omega$ for Case $1,2.4 \mathrm{~m} \Omega$ for Case 2 , and $2.3 \mathrm{~m} \Omega$ for Case 3 . The change of line resistance is less dependent on the bath height than the bath radius.

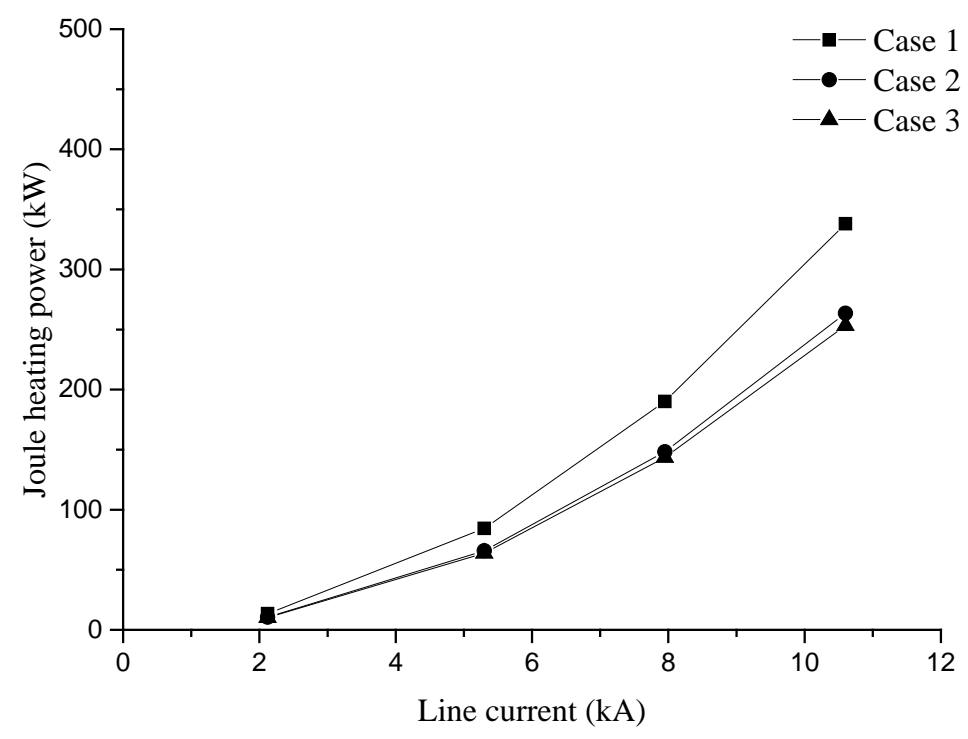

Fig. 4. The Joule heating power variation with the line current (RMS) for the three cases.

\section{Summary}

A 3D numerical model based on finite element method was developed for the analysis of MHD mixed convection of the melt in the EFMF. The electromagnetic field was obtained by 3-D harmonic edge-based analysis. The measured magnetic field agreed well with the calculated results. The combined effects of forced and natural convection caused by electromagnetic stirring and buoyancy were both considered because the Richardson number is approximately equal to unity. The convection was found to be fully turbulent in three typical cases of melting process, and the Reynolds number increases with larger line current or smaller bath volume. The predicted temperature and flow field revealed that the average temperature field is significantly influenced by the electromagnetic stirring, and the difference of the bath temperature is less than $50 \mathrm{~K}$ in the cases. The quantitative 
analysis of the line resistance indicates that it is more easily influenced by the bath radius than the bath height.

\section{Acknowledgements}

This work is supported by National Natural Science Foundation of China [Grant No. 51604059]; International Science \& Technology Cooperation Program of China [Grant No. 2014DFR50880]; and the Fundamental Research Funds for the Central Universities [Grant No. DUT16QY35].

\section{References}

[1] X. Zhang, D.F. Xue, D.L. Xua, X.Q. Feng, and J.Y. Wang: Growth Vol. 280 (2005), p. 234-238

[2] X. Zhang, D.F. Xue, J.Y. Wang, and X.Q. Feng: J. Cryst. Growth Vol. 292 (2006), p. 505-509

[3] U. Ortiz, J. Aguilar, C. Esparza, and C. Guerrero: Mater. Sci. Eng. Vol. 5: (1997), p. 347-356

[4] Y. Zhang, and S. Li: IEEE Trans. Ind. Informat. Vol. 9(4) (2013), p. 2248-2255

[5] Y. Zhang, and S. Li: Control Eng Pract. (2014), p. 194-204

[6] Y. Fan, W. Du, Y. Zhang, and X.G. Wang: Math. Probl. Eng. (2015), p. 1-15

[7] G. Caffery, D. Warnica, N. Molloy, and M. Lee: Temperature Homogenisation in an electric arc furnace steelmaking bath. Proceedings of the International Conference on CFD in Mineral and Metal Processing and Power Generation. CSIRO, (1997), p. 87-99

[8] F. David, T. Tudorache, and V. Firteanu: Numerical evaluation of electromagnetic field effects in electric arc furnaces. COMPEL: Int. J. for Computation and Maths. in Electrical and Electronic Eng. Vol. 20: (2001), p. 619-635

[9] A. Kiyoumarsi, A. Nazari, and M. Ataei: Electromagnetic analysis of an AC electric arc furnace including the modeling of an AC arc. COMPEL: Int. J. for Computation and Math. in Electrical and Electronic Eng. Vol. 29: (2010), p. 667-685

[10] N. Arzpeyma, O. Widlund, M. Ersson, and P.G. Jonsson: ISIJ Int. Vol. 53(1) (2013), p. 48-55 [11] Z. Wang, Y. Fu, N.H. Wang, and L. Feng: J. Mater. Process. Technol. Vol. 214(11) (2014), p. 2284-2291.

[12] A.L. Leu, S.M. Ma, and H. Eyring: Properties of molten magnesium oxide, Proceedings of the National Academy of Sciences of the United States of America Vol. 72 (1975), p. 1026-1030 